\title{
Apprendre, un moment de vie ? Savoir, expérience et rapport au vivant
}

\author{
Julie Noack
}

CERPHI, Ecole Normale Supérieure de Lyon - 15, Parvis René Descartes, 69007, Lyon, France Département d'Anthropologie, Université de Montréal - 2900, Boulevard Edouard-Montpetit, Montréal, QC H3T 1J4, Canada

\begin{abstract}
Résumé. L'objectif de cette communication est de comparer sur le plan pédagogique deux conceptions du rapport entre science et vivant, diamétralement opposées sur le plan épistémologique. En se basant sur un livre [1], une conférence [2] et un entretien [3], on analysera l'expérience de pensée par laquelle le biologiste et épistémologue Jean-Jacques Kupiec fait se sentir vivants les apprenants afin de mieux les conscientiser aux réquisits de la démarche scientifique. On comparera ensuite les motivations de Kupiec avec les idées pédagogiques du philosophe-médecin Georges Canguilhem (1904-1995) afin d'essayer de comprendre pourquoi l'un et l'autre jugent pertinent de faire remarquer à l'apprenant qu'il est actuellement en vie. Car, malgré des conceptions du vivant diamétralement opposées, Canguilhem et Kupiec se rejoignent sur l'idée que l'expérience vitale joue un rôle crucial - que ce soit en positif ou en négatif, comme un obstacle épistémologique ou comme un levier heuristique - dans l'apprentissage et la pratique de la biologie ou de la médecine. Prendre en charge cette expérience constitue peut-être une ressource face au double défi (scientifique et éthico-politique) qui échoit à l'éducation aux sciences du vivant.
\end{abstract}

\begin{abstract}
Is learning part of living - and vice versa? Canguilhem and Kupiec on experience, knowledge and the perception of life. This paper compares the views of Georges Canguilhem (philosopher of medicine and physician) regarding education in the life sciences with the way Jean-Jacques Kupiec - biologist and epistemologist at the École Normale Supérieure (Paris) - concretely teaches through his talks and books. My point is to try to understand why both Kupiec and Canguilhem consider that it is relevant to draw the attention of the listener (or the reader) on the fact that he himself is a living organism. By focusing on the thought experiment Kupiec uses to relate the notion of epigenetics to the problem of individual existence, I try to show that (despite completely opposite views on the nature of life) Canguilhem and Kupiec agree on the fact that feeling alive plays a significant part in learning biology or medicine. Whether it is as an obstacle to overcome or as an intuition to explore, our bodily experience of organic functioning has to be dealt with whenever we are studying life sciences or introducing people to them.
\end{abstract}




\section{Introduction}

\subsection{Le contexte : un double défi pour l'éducation aux sciences du vivant}

Comme le souligne Michel Morange (2008) [4], Canguilhem semble un passage obligé dans la formation médicale alors même que les données scientifiques dont il parle sont datées. Mais si, en 2015, on y utilise des ouvrages qui remontent à 1943 et 1952, il n'y a là aucun paradoxe - poursuit M. Morange. Ce qui serait en effet impensable dans un département de biologie (où un livre n'est plus ouvert dès qu'il a dix ans d'âge) est par contre tout à fait normal en faculté de médecine - $d u$ fait $d u$ double défi constitutif de la formation spécifique qui y est proposé ${ }^{1}$. Prenons le temps d'expliciter ce double défi en développant le résumé que fait M. Morange des conceptions de Canguilhem: La première finalité de l'éducation aux sciences du vivant est que l'apprenant intègre les modèles objectifs du fonctionnement organique. Le but est de convaincre rationnellement l'apprenant que les modèles explicatifs présentés par l'enseignant sont plus dignes de valeur que les croyances ou les impressions subjectives qu'il peut avoir de la manière dont fonctionne son corps. Ainsi, si les premières années du cursus médical sont si difficiles, c'est en partie parce qu'on y apprend à «objectiver » (comme on dit) - c'est-à-dire à nier, le temps de la dissection d'un cadavre ou d'un diaporama d'anatomie pathologique, le savoir subjectif que constitue notre vécu, notre expérience du corps propre. «Objectiver », c'est remplacer les représentations subjectives (personnelles) par des représentations objectives (impersonnelles) : désinvestir émotionnellement la situation passe donc par le fait de se concentrer sur cette même situation (plutôt que de détourner l'attention en pensant à autre chose) mais vue du "point de vue de nulle part» (Nagel, 1986 [5]) que constitue sa projection dans des coordonnées objectives (un référentiel où je ne suis pas présent) ${ }^{2}$.

Mais la seconde finalité de l'éducation aux sciences du vivant est que l'apprenant soit initié aux problèmes éthiques et politiques liés au vivant (Canguilhem, 1972 [6], p.7) toujours sur la base d'une réflexion rationnelle. En effet, contre une tentation irrationaliste exploitée par les idéologies, il s'agit de répondre aux attentes de l'apprenant ${ }^{3}$ - c'est-à-dire aux questions que, du fait de sa pratique mais aussi des problèmes de société, il se pose ou sera amené à se poser - pour éviter que, déçu par une objectivation unilatérale qu'il considérerait comme purement théorique, il radicalise des intuitions non-questionnées en embrassant autant de discours tout faits. Or c'est là un vrai défi car, sur les questions de «vie » et «vivant», les idéologies profitent du caractère autocritique et volontairement

\footnotetext{
${ }^{1}$ Sauf mention contraire dans le cas de citations, c'est nous qui soulignons les expressions en italiques.

${ }^{2}$ Par l'expression de " view from nowhere », le philosophe Thomas Nagel désigne la capacité de se représenter mentalement sa propre activité « en $3^{\mathrm{e}}$ personne » (c'est-à-dire telle que la percevrait un observateur extérieur) alors qu'on est encore en pleine action. Expliquant que l'objectivité scientifique porte cette capacité à son paroxysme, il affirme que l'expérience phénoménologique (ce qui est vécu «en $1^{\text {ère }}$ personne ») est porteuse d'informations qui sont tout à la fois essentielles à la compréhension de l'activité en question et inaccessibles en dehors du point de vue (personnel et situé) de l'acteur qui accomplit cette action. Plutôt que d'aborder cette "querelle du réductionnisme" en général et pour elle-même, nous voudrions discuter ici ce que change concrètement ce débat sur le plan pédagogique dans le cas des sciences du vivant. Si l'épistémologie de J.-J. Kupiec est radicalement objectiviste et celle de Canguilhem résolument phénoménologique (au point de se dire vitaliste), notre but est simplement de montrer que dans les pratiques pédagogiques l'un comme l'autre recourent à un procédé rhétorique (proche de l'expérience de pensée) qui consiste justement dans l'aller-retour entre ces deux positions : faire ressentir à l'auditeur le vécu de l'objectivation tout en lui présentant ce «décentrement » comme le principe essentiel de la démarche scientifique lorsqu'elle prend pour objet le vivant.

${ }^{3}$ Nous utilisons ce terme pour désigner, sans distinction, l'élève et la personne participant aux activités de médiation scientifique, pour insister sur le point commun à leurs activités respectives. Canguilhem parle plus généralement d' « homme vivant» (2000 [7], p.196) afin de souligner que «l'homme savant» (idem) - au double sens du chercheur et de l'apprenant - partage avec l'organisme une même activité de "recherche inquiète » (1995, [8], p.364) qui est « recherche de la sécurité par réduction des obstacles [et] résolution directe ou indirecte des tensions entre l'homme et le milieu» (2000 [7], p.12).
} 
limitatif de la science pour instrumentaliser celle-ci. En effet, se présentant comme un «supplément d'âme » (ou «conscience » surajoutée à la science afin qu'elle se soit pas « ruine de l'âme ») ou comme une «compréhension intuitive» compassionnelle (par opposition à «l'explication» discursive neutre, purement rationnelle), cet irrationalisme réussit le tour de force de se présenter comme complémentaire - et même comme le complément nécessaire - par rapport à l'analyse scientifique de la réalité. Or le cynisme atteint ici son comble, puisque ce faisant, ces idéologies font exactement l'inverse du but recherché par l'éducation aux sciences du vivant : elles instillent l'idée que les problèmes éthico-politiques liés au vivant ne seraient qu'une question d'opinion, qu'une affaire de convictions personnelles - c'est-à-dire de croyances qui n'auraient finalement aucun compte à rendre vis-à-vis de la réalité que nous font connaître les sciences.

Si l'œuvre de Canguilhem reste un incontournable de la formation médicale, c'est selon nous parce qu'elle a été explicitement écrite pour se poser la question de la relation entre ces deux défis : connaître scientifiquement le vivant, d'une part, et fonder le débat rationnel où prendre en charge les problèmes éthico-politiques liés au vivant sur les résultats et sur la démarche des sciences du vivant, d'autre part. Le souci fondamental de Canguilhem ${ }^{4}$ est en effet d'essayer de comprendre comment d'un côté ces deux défis paraissent être mutuellement exclusifs, alors que d'un autre côté ils ne peuvent pas être séparés sans devenir contre-productifs (par rapport aux buts du projet pédagogique global de l'enseignement et de la médiation en sciences du vivant).

\subsection{Le fil rouge : est-il ou non pertinent de faire remarquer aux apprenants ce fait : " apprendre est un moment de vie »?}

Nous aimerions nous demander si - en situation d'enseignement ou de médiation scientifique dans le cadre des sciences du vivant - il est pertinent (ponctuellement et à un moment bien précis) d'attirer l'attention de l'apprenant sur le fait que lui aussi est un être vivant en situation de vie, qu'apprendre (comme il le fait) est un acte qui s'inscrit dans le prolongement d'une activité vitale qu'il a en commun avec l'organisme qu'il étudie - bref : lui faire remarquer le fait que «apprendre est un moment de vie » au double sens où l'on reste vivant quand on apprend et où une composante de l'activité «vivre » consiste dans le fait d'apprendre (de découvrir son corps, l'environnement et les possibilités qu'offre leur interaction) ${ }^{5}$.

La question peut sembler étrange, car elle mélange deux choses qui n'ont a priori rien à voir : les modèles scientifiques et le vécu corporel. Or il n'y a - semble-t-il - aucun intérêt à mentionner une coïncidence aussi anecdotique que le fait que «sujet» et «objet» des sciences du vivant ont pour point commun d'être «vivants». Pire : vouloir attirer l'attention sur ce détail hors-sujet brouille le discours d'objectivité et risque d'ouvrir la porte à des réactions et à des commentaires personnels qui n'ont tout simplement pas leur place dans le cadre de l'éducation aux sciences du vivant.

Pour radicaliser cette objection, nous allons l'incarner dans un exemple concret de diffusion scientifique : les productions «grand public» de J.-J. Kupiec. Difficile, a priori, de choisir un travail qui soit plus opposé à celui de Canguilhem : J.-J. Kupiec défend en effet avec véhémence une vision strictement matérialiste du vivant - là où, face aux soupçons de «vitalisme » (au sens, classique, de théorie métaphysique explicative), Canguilhem répond en redéfinissant le vitalisme comme l'exigence

\footnotetext{
${ }^{4}$ «Comment résoudre cette difficulté : si on se place au strict point de vue objectif, il n’y a pas de différence entre la physiologie et la pathologie ; si on cherche dans les valeurs biologiques une différence entre elles, on a quitté le terrain scientifique? Nous proposerions comme éléments d'une solution les considérations suivantes. » (1972 [6], p.149).

${ }^{5}$ Pour lever l'ambiguïté en distinguant deux expressions, on dira dans le premier cas que «apprendre, c'est un moment de vie » et, dans le second, que "un moment de la vie, c'est d'apprendre» - en prenant (comme Canguilhem, 1994 [8], p.335) le terme de vie au sens biologique et non au sens existentiel.
} 
de reconnaître l'originalité spécifique du vivant (2000 [7], p.86-87) pour s'en revendiquer, non sans provocation vis-à-vis des réductionnistes persuadés qu'il n'y a pas débat ${ }^{6}$.

Or notre question de départ fait elle aussi débat, elle se discute, malgré sa formulation un peu naïve. Pour «mettre en scène » ce débat, nous allons partir du problème que pose l'objection (le refus d'un "mélange des genres"), pour essayer de montrer en quoi tous ces aspects sont autant de raisons négatives pour lesquelles il serait utile de thématiser, à certains moments stratégiques de la situation pédagogique, cette situation de réflexivité si particulière. Dans le second temps du texte, nous suggérerons en quoi la possibilité de retourner ce problème en opportunité constitue une raison positive de mentionner le fait que les processus d'apprentissage font partie intégrante de l'activité organique : amener l'apprenant à réfléchir aux implications possibles de cette réalité peut en effet constituer une ressource intéressante pour fonder la discussion rationnelle des problèmes de « bioéthique » (l'utilisation des cellules souches, la fin de vie, le diagnostic préimplantatoire...) et de «biopolitique »- au sens strict (la biométrie, la surveillance épidémiologique, le planning familial...) et au sens large (les OGM, le réchauffement climatique, la biodiversité...) - sur la plus grande continuité possible avec les résultats et la démarche des sciences du vivant.

\section{Les effets négatifs dus au fait qu'apprendre reste un moment de vie peuvent être en partie pris en charge à travers une conscientisation ponctuelle critique.}

Les concepts de biologie auxquels Kupiec veut introduire le public sont pour partie traditionnels et pour partie novateurs (voire iconoclastes). Pour présenter les concepts d' «ontogenèse» et de «différenciation cellulaire » de la biologie moléculaire, l'orateur part d'une question : «par quel genre de mécanismes explique-t-on la transformation de la cellule-œuf en un organisme tridimensionnel, différencié et opérationnel ?». Pour dramatiser l'enjeu, il fait immédiatement remarquer que ce développement embryonnaire est un processus très fiable alors qu'il est très complexe : le public est donc invité à s'étonner de ce qu'il n'y ait statistiquement pas plus d'échecs dans ce processus d'embryogenèse. C'est pour répondre à cet étonnement que Kupiec présente alors au terme d'un historique (2008 [1], p.63) - la façon contemporaine de répondre à cette question : l'expression des gènes qui va entraîner la différenciation cellulaire (donc la formation de l'organisme à partir des «plans » de son génotype) est régulée par les gènes eux-mêmes. Autrement dit (Kupiec, 2015 [3], p.275), le processus d'embryogenèse est conçu comme un processus déterministe au sens où chaque événement y répondrait à une «loi »-même si dans ce cas précis, une telle «loi » n'est plus conçue sur le modèle d'une loi physique mécaniste mais sur le modèle d'une «instruction» dictée par un « programme » «à réaliser».

C'est à partir de ce point que les concepts introduits par Kupiec (1983) [10] deviennent originaux, puisqu'il s'agit d'une interprétation radicale du concept d'épigénétique fondée sur des travaux de recherche que l'auteur lui-même mène depuis le début des années 1980. Si l'on définit l'épigénétique comme l'idée selon laquelle l'expression des gènes est modulée par les conditions environnementales $^{7}$, on pourrait résumer la thèse de Kupiec en ces termes: les mécanismes épigénétique sont la seule façon dont l'expression des gènes est régulée. Baptisant cette théorie « darwinisme cellulaire»(2008 [1], p.184-199), Kupiec affirme ainsi que « en réalité tous les gènes "fuient" un peu : il n'y a jamais de gène qui soit totalement éteint. Tous les gènes s'expriment à un très, très bas niveau, et il y en a certains qui s'expriment fortement. Peut-être donc qu'à partir de là on peut avoir une vision sélective » (2015 [3], p.274). Une telle «vision sélective » consiste dans l'idée

\footnotetext{
${ }^{6}$ Kupiec et Canguilhem ont en commun d'affirmer avec force que les sciences du vivant sont aussi bien l'objet de débats que (plus important encore) le lieu de débats (Kupiec 2013, [9]).

${ }^{7}$ Environnement de l'organisme (son milieu, les conditions de vie extérieures) mais aussi environnement de telle cellule de cet organisme (le tissu de tel organe dont elle est une partie).
} 
que d'une part c'est une différence de degré qui va distinguer à un moment donné le gène qui s'exprime et celui qui ne s'exprime pas - et d'autre que ce qu'on appelle aujourd'hui les « voies de signalisation» ne doit pas être conçu autrement que les caractères phénotypiques hérités de la phylogenèse (la structure cellulaire, par exemple). Autrement dit, la régulation de l'expression des gènes serait un phénomène statistique (dû aux différences dans l'importance quantitative des interactions) où les différences seraient dues à des mécanismes de compartimentation dans la cellule. Or l'intérêt principal de cette généralisation de l'idée d'épigénétique est de remplacer le dualisme ${ }^{8}$ entre la causalité aveugle («hasard-sélection») qui a lieu à l'échelle de la phylogenèse et le la causalité de type «programmiste » qui a lieu (dans la vision actuelle) au niveau de l'ontogenèse - et le remplacer par une vision unitaire : la très grande stabilité du développement embryonnaire est de même nature que la très grande stabilité de la reproduction inter-générationnelle (l'une et l'autre comportant une marge de variation irréductible).

Maintenant que nous avons aperçu les idées que Kupiec juge important de transmettre au public, concentrons-nous sur la façon dont il présente les choses et sur les raisons pour lesquelles il considère qu'il est urgent de sensibiliser à ces questions-là (plutôt qu'à d'autres) les personnes qui n'ont pas spécialement de contact avec le monde de la recherche. Par souci de précision, nous concentrerons l'analyse sur une seule occurrence d'une triple opération que l'auteur fait systématiquement en dehors de ses publications scientifiques de pointe - à savoir : 1) attirer l'attention du lecteur/auditeur sur le fait que la relativisation des évidences vécues crée chez lui un malaise, 2) lui expliquer que ce malaise est le signe d'une résistance à l'objectivation (d'une survivance des préjugés), et enfin 3) lui présenter le dépassement de tels biais cognitifs et psychologiques comme le moteur de la démarche scientifique.

«Dictyostelium discoïdeum est un protiste eucaryote qui peut vivre sous forme d'amibe unicellulaire lorsque les conditions environnementales sont favorables mais, lorsque ces conditions s'appauvrissent, ces amibes s'agrègent et se différencient en deux types cellulaires. Elles forment alors un petit champignon multicellulaire qui engendre des spores qui - à leur tour - donnent naissance à de nouvelles amibes unicellulaires, lorsque l'environnement redevient favorable.

Dans ce cycle, où est l'individu: les amibes unicellulaires, le champignon multicellulaire, ou les spores? Encore plus problématique : où est l'origine dans ce processus ? On est confronté à une alternance continue de phases unicellulaires et multi-cellulaires qui se poursuit indéfiniment en fonction des conditions environnementales. Il n'y a ni origine, ni fin précise dans ce processus.

En ce qui nous concerne, nous sommes persuadés que nous, les humains, n'avons rien à voir avec ce petit champignon, parce que nous sommes bien plus sophistiqués. Nous sommes convaincus qu'un processus réel d'embryogenèse nous construit. Il aurait une origine évidente (l'œuf fécondé fait de nos cellules germinales) et une fin, un stade terminal tout aussi évident : l'individu adulte c'est-à-dire nous-mêmes.

Il s'agit d'une projection anthropocentrique typique. Avec du recul, nous pouvons nous apercevoir aisément que dans le cas des humains nous avons aussi affaire - comme pour Dictyostelium - à une alternance continue de phases unicellulaires (correspondant aux cellules germinales) et multicellulaires (correspondant aux êtres adultes). Ce processus est continu, puisque l'œuf fécondé est fait de cellules germinales parentales qui passent dans la descendance. Il y a en nous des éléments cellulaires (et pas

\footnotetext{
${ }^{8}$ Ce dualisme repose, on le voit, sur l'idée d'une séparation absolue entre l'échelle de l'évolution des espèces («phylogenèse », échelle qui est celle des mutations du génome), et l'échelle des organismes individuels (du développement embryonnaire, l'« ontogenèse », et du fonctionnement physiologique).
} 
seulement de l'ADN) provenant de nos parents. Il n'y a pas de discontinuité mais une continuité faite de cycles cellulaires qui traversent les générations.

Nous sommes spontanément finalistes. Nous nous plaçons de notre point de vue et nous sommes persuadés que ce qui est important, dans l'alternance des phases unicellulaires et pluricellulaires, c'est ce que nous appelons l'embryogenèse. Nous sommes persuadés qu'il s'agit d'un processus objectif qui est là pour nous construire. Mais avec du recul, nous voyons que ce point de vue est subjectif, et qu'il privilégie la phase qui va de l'unicellulaire au multicellulaire parce que cela fait de nous la finalité des processus biologiques. En réalité, notre regard isole cette phase dans le processus continu. Si nous étions des cellules germinales, verrions-nous les choses de cette manière? Il est probable que dans ce cas, nous privilégierions la phase qui va du multicellulaire à l'unicellulaire. Du point de vue des cellules germinales, ce qui est réel, ce n'est pas l'embryogenèse, mais les processus d'ovogenèse et de spermatogenèse qui permettent leur individuation.

Cela peut sembler surprenant ou un pur exercice de style, sauf que c'est à peu près le point de vue de la génétique lorsqu'elle est cohérente avec elle-même et va au bout de sa logique. » (Kupiec, 2012 [2], p.15-17)

Ayant donné une exposition minimale du paradigme du "programme génétique », l'orateur passe à l'analyse critique des présupposés de cette théorie - c'est le passage d'où est tiré l'extrait. On l'a dit, Kupiec affirme que le modèle du «programme génétique » oblige à traiter différemment les espèces (dont le devenir n'obéit à aucun ordre prédéfini) et l'individu (dont le devenir - notamment pendant l'embryogenèse - obéirait à un ordre figé, un «processus réel » unitaire). Mais alors : si c'est à cette idée que Kupiec veut parvenir, pourquoi un tel détour par le cas particulier de l'amibe Dictyostelium ? Pourquoi interrompre le fil de sa conférence par ce qui a tout l'air d'être une digression?

En fait, l'enjeu de cette expérience de pensée est décisif pour l'orateur - et c'est bien pour cela qu'il la place au début de la conférence, juste après les définitions initiales. L'épistémologue tient en effet d'emblée à thématiser le mécanisme de fonctionnement d'une illusion - c'est-à-dire d'un biais structurel $^{9}$ dans la perception et la compréhension de la réalité. Or, puisque le propre d'une illusion est de continuer à agir même une fois démentie, Kupiec ne se contente pas d'en parler : il veut faire sentir le plus directement possible au public l'effectivité d'une illusion à l'œuvre dans sa vision du monde. Il va donc chercher à provoquer une réaction affective puis une analyse réflexive de celle-ci - et c'est ce qui explique le détour par l'illustration du cas de l'amibe.

Le mécanisme d'illusion auquel l'orateur veut sensibiliser son public, c'est l'autocentrisme : notre tendance systématique à diviser et classer le monde qui nous entoure à partir de catégories dérivées de ces quelques catégories considérées comme fondamentales uniquement parce qu'elles ont été forgées pour nous convenir ${ }^{10}$. Or nous sommes tous convaincus d'être depuis longtemps guéris de ce préjugé,

\footnotetext{
${ }^{9}$ Cette définition concerne aussi bien l'illusion d'optique (comme les divergences constantes, dans le champ visuel, entre ce que perçoivent respectivement l'œil droit et l'œil gauche - des divergences qui sont la condition même de la vision en relief et que le cerveau apprend à négliger), l'illusion psycho-logique - définie par Freud (1995 [11], p.32) comme une représentation mentale dont le contenu est dicté par le rôle qu'elle joue dans le fonctionnement psychique -, et enfin l'illusion cognitive - la tendance qu'ont les schèmes de compréhension dont nous héritons à s'auto-valider en filtrant notre perception de la réalité et en pré-orientant l'interprétation des faits susceptibles de les contredire.

${ }^{10}$ Le botaniste Francis Hallé parle ainsi du «zoocentrisme» (1999 [12], p.212) de la biologie contemporaine pour avancer l'idée que les modèles fondamentaux d'explication du vivant sont calqués sur le cas des animaux, si bien que l'enseignement et la recherche en biologie végétale (champ déconsidéré du fait qu'il ne nous apprend rien sur l'humain) sont constamment freinés par la projection de concepts mal adaptés à ce qui fait la spécificité des phénomènes qu'on y étudie. La différence entre «métazoaires » et « eumétazoaires 》 (littéralement les vrais animaux, aussi appelés d'ailleurs métazoaires supérieurs) relève du même biais cognitif : les éponges (qui font
} 
puisque nous nous moquons avec condescendance de la naïveté géocentriste et nous nous indignons de l'obscurantisme anthropocentriste qui refusait catégoriquement l'idée que l'homme soit une espèce qui puisse descendre d'une autre. En commençant sa conférence, Kupiec envoie donc un message très clair : être convaincu que l'autocentrisme, c'est le tort des autres (le Moyen-âge, le XIX siècle, etc.), c'est le comble de l'illusion autocentriste, et c'est là que doit se concentrer l'effort d'apprentissage (et de recherche) de n'importe quelle science. Avant d'apprendre les contenus (le modèle du programme génétique par exemple) c'est d'abord ce travail sur soi qu'il faut exercer comme une habitude car sans ce principe essentiel de la démarche scientifique, la science se retourne littéralement en son contraire.

Pour que son expérience de pensée ne prenne pas le public au dépourvu, Kupiec prend le soin de le prévenir en annonçant d'emblée là où il veut en venir :

«La notion d'individu est aussi problématique que celle d'espèce. Nous en avons tous une idée qui semble évidente : il s'agirait d'une unité morphologique et fonctionnelle. Cependant il est tout aussi évident que nous avons forgé cette idée de l'individu en nous regardant nous-mêmes, ou les vivants qui nous sont très proches. » (Kupiec 2012 [2], p.14).

S'ensuivent donc l'image de Dictyostelium et le passage cité. On veut analyser le ressort affectif de cette présentation, mais pour être parfaitement clair sur les enjeux que l'auteur met lui-même dans ce procédé, éclairons l'extrait de conférence par un extrait de livre où Kupiec thématise le problème :

«La science se construit progressivement en se dégageant de l'évidence du sens commun. L'espèce est une évidence, mais une évidence métaphysique, y compris en biologie. Si l'on a du mal à s'en débarrasser, c'est probablement beaucoup plus par anthropocentrisme que pour des raisons logiques. En effet, s'il nous est relativement acceptable que l'espèce soit une notion arbitraire quand il s'agit des montagnes et des rivières, cela devient beaucoup plus dangereux quand il s'agit d'animaux car on se rapproche de l'homme, et qu'inévitablement on en viendra à questionner également sa spécificité. Douter de notre existence objective en tant qu'espèce nous est évidemment impensable et insupportable. Cependant, si notre objectif est de construire une théorie rationnelle de la biologie, nous sommes obligés d'analyser calmement cette question.» (Kupiec, 2000 [13], p.35)

Le lexique de l'affect - « insupportable », « calmement » - annonce déjà que la difficulté («avoir du mal à se débarrasser » des "évidences du sens commun») à adopter une démarche scientifique à propos de soi-même vient de la vie corporelle et/ou psychique, et non pas « de raisons logiques ». En ce sens on comprend bien pourquoi, dans la conférence, l'orateur va jouer sur les affects du public : il veut très précisément lui faire reconnaître «calmement» qu'il y a quelque chose d' «insupportable » dans la connaissance biologique. Le but final est de conclure que, tant que l'on n'aura pas reconnu ce biais et affronté cette question dérangeante, on restera prisonnier d'un préjugé anti-scientifique. C'est la raison pour laquelle l'ouvrage cité à l'instant se conclut par l'ouverture suivante :

«Chassé de la nature extérieure par Copernic et Darwin, l'anthropocentrisme s'est réfugié à l'intérieur de nous-mêmes. Ce repli semble inexpugnable. Pouvait-on imaginer que nous n'étions pas le centre, ni la finalité de notre propre individu ? C'est pourtant le cas. » (Kupiec, 2000 [13], p.213-214).

partie du premier groupe mais pas du second) sont ici classées à partir de ce qu'elles ne sont pas, car c'est l'homme classificateur qui porte avec lui le référentiel (ce qu'est "vraiment" un animal, quel critère départage le "supérieur" de l'inférieur, etc.). 
Difficile de ne pas songer, en lisant ces lignes, au célébrissime texte de Freud sur les «blessures narcissiques » (1961 [14], p.266-267) infligées par la science à «l'égoïsme naïf de l'humanité » croyant être planète au centre de l'univers, espèce au sommet de la création et individu « maître dans sa propre maison ». Car il est significatif que, quand Kupiec explicite le geste épistémologique qu'il est en train d'accomplir, il reprenne littéralement la rhétorique de ce texte, en se présentant lui-même à la place que s'attribuait Freud. C'est que, alors même que Kupiec et Freud n'ont absolument rien en commun, leur argument précis est ici exactement le même : l'objectivité scientifique est un effort qui consiste à mettre au jour et à neutraliser les biais structurels de notre connaissance pré-scientifique.

Kupiec est loin d'être freudien, mais justement : l'appel qu'il fait aux affects n'en est que plus intéressant. En effet, on a beau être rationaliste militant, la nature de l'illusion autocentriste est telle qu'on ne saurait se contenter de l'analyser rationnellement : il faut faire en sorte que le public luimême puisse toucher du doigt le biais qui est actuellement en train de fausser sa compréhension.

Si l'auditeur est invité ici d'abord à se regarder lui-même comme s'il était une amibe puis à se mettre en pensée à la place d'un gamète pour regarder de là ce qu'il est actuellement, c'est donc bien pour s'essayer à un exercice qualifié d' «impensable et insupportable » alors qu'il ne s'agissait que du concept $d^{\prime}$ ' «espèce humaine ». Combien cet exercice est-il encore plus «insupportable » quand il s'agit de douter cette fois de notre individualité dont un concept essentialiste garantit la sacralité. Nous avons fini par accepter - dit en somme l'orateur - que l'homme descend du singe parce que, finalement, la distance temporelle est si grande que nous pouvons nous convaincre que cela n'a plus rien à voir avec nous. Par contre, la même opération de relativisation devient extrêmement dérangeante quand elle touche notre individualité corporelle, c'est-à-dire notre intimité : on nous invite à nous vivre (un instant) comme si nous n'étions qu'un processus de maturation de nos gamètes ${ }^{11}$.

En faisant sentir au public qu'il est juge et partie dans une discussion sur l'individualité biologique, l'orateur veut provoquer un certain effet cognitif. Lequel ? Qu'est-ce qui lui paraît important d'échanger dans la médiation scientifique où il s'investit - et pourquoi ?

L'insistance de Kupiec sur le fait que l'anthropomorphisme (qui trompe aussi bien le nonscientifique que le généticien) est un biais nécessaire correspond exactement à la définition de «l'illusion» donnée par Freud: une représentation ou une croyance qui (indépendamment du fait qu'elle soit vraie ou fausse ${ }^{12}$ ) est dictée par un besoin, par un rôle dans le fonctionnement du psychisme. L'illusion est une idée structurellement nécessaire à l'individu pour vivre.

Dans le fameux texte des «blessures narcissiques », Freud inscrit toutes les grandes avancées de la science dans la continuité d'un effort à travers lequel la rationalité scientifique parvient à «gagner sur » la résistance de nos illusions. Nous avons besoin, pour vivre, d'agir comme si la Terre était immobile $^{13}$, d'agir comme si la dignité de «personne » revenait à l'humain en vertu d'une différence essentielle avec les autres êtres vivants, d'agir comme si notre conscience réfléchie avait une maîtrise sur notre psychisme, d'agir enfin comme si notre individualité biologique avait une existence réelle, objective. « Nous sommes spontanément finalistes », dit Kupiec, mais c'est exactement pour la même raison que nous sommes aussi spontanément (dans notre façon de nous vivre au quotidien) géocentristes, fixistes et cartésiens. Or Kupiec et Freud ne sont pas seulement d'accord sur le diagnostic, ils le sont aussi sur la thérapie : il faut que la vision scientifique du monde parvienne à relativiser cette apparence trompeuse de centralité absolue (notre conscience s'imagine être centre de

\footnotetext{
${ }^{11}$ La même expérience de pensée, en vue du même effet - quoique pour appuyer une thèse diamétralement opposée - constitue le premier chapitre («Why are people?») d'un célèbre livre de Richard Dawkins (2006 [15]).

${ }^{12}$ «Une illusion n'est pas la même chose qu'une erreur, elle n'est d'ailleurs pas nécessairement une erreur. » dit bien Freud (1995 [11], p.31).

${ }^{13}$ Voir la célèbre analyse de Husserl à ce sujet (1989 [16]).
} 
l'univers, centre de la création, centre du psychisme, centre de la lignée généalogique) en projetant ce que nous sommes (notre planète, notre espèce, notre vie psychique, notre vie organique) dans des coordonnées objectives - dans un référentiel neutre, universel, parce que conventionnel : un modèle d'univers en expansion accélérée, un graphe phylogénétique en cercle (plutôt qu'en arbre), une "hypothèse $e^{14}$ d'un inconscient psychique », une succession indéfinie de cycles alternant phases unicellulaire et pluricellulaire (la lignée généalogique) où le point de vue découpe arbitrairement le début et la fin de «l'individu».

Gaston Bachelard a particulièrement travaillé cette question de l'illusion et du rôle irremplaçable de la rationalité scientifique pour sortir la représentation du monde des illusions où elle est initialement prise. En termes bachelardiens, en effet, le procès même de la science est une entreprise d' « objectivation » (1966 [18], p.53) - c'est-à-dire un effort de «rupture » contre la fausse évidence tenace des «obstacles épistémologiques » (que sont tous les préjugés cognitifs que nous héritons de l'expérience commune) grâce à la critique qui relativise les impressions subjectives à leurs conditions objectives. Bachelard ne s'étant intéressé qu'aux sciences physiques et chimiques, c'est Canguilhem qui a «exporté » l'épistémologie bachelardienne sur le terrain des sciences du vivant, tandis que Bourdieu l'a fait sur celui des sciences humaines ${ }^{15}$. Car, si les trois illusions sont identiques quant à leur fonctionnement, chacune est suffisamment spécifique pour devoir être prise en charge par une science différente. La physique mathématique, selon Bachelard, doit se construire contre les illusions d'une «physique naïve»; la sociologie, selon Bourdieu, doit se construire contre la «petite sociologie spontanée » que produit en nous notre expérience du monde social. Quant à la biologie :

« Le milieu dont l'organisme dépend est structuré, organisé par l'organisme lui-même. Ce que le milieu offre au vivant est fonction de la demande. [...] En sorte que l'environnement auquel il est censé réagir se trouve originellement centré sur lui et par lui.

Mais l'homme (en tant que savant) construit un univers de phénomènes et de lois qu'il tient pour un univers absolu. La fonction essentielle de la science est de dévaloriser les qualités des objets composant le milieu propre, en se proposant comme une théorie générale d'un milieu réel c'est-à-dire inhumain. Les données sensibles sont disqualifiées, quantifiées, identifiées. L’imperceptible est soupçonné, puis décelé et avéré. Les mesures se substituent aux appréciations, les lois aux habitudes, la causalité à la hiérarchie, et l'objectif au subjectif. » (Canguilhem, 2000 [7], p.153)

Pour Canguilhem, l'obstacle épistémologique spécifique aux sciences du vivant est la nécessité vitale pour l'organisme - de se vivre comme le «centre »d'un «milieu ${ }^{16}$. Au contraire, les sciences du vivant montrent quels sont les mécanismes par lesquels l'environnement (les conditions objectives) détermine des réactions en chaîne à l'intérieur du fonctionnement organique. Entre ces deux «visions» de la même réalité, on retrouve exactement la même opposition que dans les configurations précédentes du conflit entre illusion et objectivité. Dans l'épistémologie

\footnotetext{
14 «Qu'il soit justifié d'admettre un psychique inconscient et de travailler scientifiquement à l'aide de cette hypothèse nous est contesté de nombreux côtés. Nous pouvons alléguer a contrario que l'hypothèse de l'inconscient est nécessaire et légitime » (Freud, 2012 [17], p.130).

${ }^{15}$ «J'ai essayé de transposer sur le terrain des sciences sociales toute une tradition épistémologique représentée par Bachelard, Canguilhem, Koyré par exemple, et mal connue à l'étranger. » (Bourdieu, 2005 [19], p.vii).

${ }^{16} \mathrm{~S}$ 'il emprunte souvent à Bachelard le concept d'obstacle épistémologique, Canguilhem va le préciser dans le cas des sciences du vivant en parlant d'«illusions ou erreurs vitales». cf. «En tant que milieu propre de comportement et de vie, le milieu des valeurs sensibles et techniques de l'homme n'a pas en soi plus de réalité que le milieu propre du cloporte ou de la souris grise. La qualification de réel ne peut en rigueur convenir qu'à l'univers absolu [...] avéré par la science, dont la reconnaissance comme tel s'accompagne nécessairement de la disqualification au titre d'illusions ou d'erreurs vitales de tous les milieux propres subjectivement centrés, y compris celui de l'homme. » (Canguilhem, 2000 [7], p.196).
} 
bachelardienne, la «blessure narcissique» n'est plus l'exclusivité des grandes découvertes, des renversements de paradigme - c'est au contraire le quotidien du scientifique, car c'est l'opération heuristique qu'il répète à chaque fois qu'il se remet à l'esprit son problème. Rien d'étonnant donc à ce que Kupiec essaie de faire vivre un instant au public ce qu'apprend à gérer le jeune étudiant en médecine - le fait de voir objectiver son propre corps. C'est qu' une telle «gymnastique » cognitive est si importante - si centrale dans la formation de l'esprit scientifique - qu'elle constitue, pour la médiation scientifique, un «essentiel» à «faire passer» de façon encore plus urgente que les contenus conceptuels.

Parler d'obstacle épistémologique dans l'apprentissage ${ }^{17}$ signifie qu'avant d'être invité à douter des évidences de sens commun (sur l'électricité, la vie, la société), l'apprenant projette des modèles d'intelligibilité biaisés sur les concepts qu'on lui présente. La métaphore du programme génétique nous paraît être sur ce point un cas d'école : à moins d'être sensibilisé en amont aux dangers d'une lecture littérale, comment l'apprenant ne sauterait-il pas sur l'occasion de comparer l'organisme à un vaste et complexe ordinateur ? C'est ce qui explique sans doute la véhémence de Kupiec: toute la ligne argumentative de $\mathrm{Ni}$ Dieu ni gène consiste à montrer que le «modèle instructeur» sur lequel repose le paradigme du programme génétique revient à une vision finaliste (dualiste et essentialiste) contraire à la démarche scientifique elle-même dans la mesure où il répète un obstacle épistémologique. C'est donc d'abord une «question de principe» sur laquelle se mobilise l'orateur pour sensibiliser son public: que l'on retienne ou non la théorie épigénétique, l'essentiel est de comprendre pourquoi la démarche scientifique exige que «les lois de la physique et de la biologie [soient] de même nature » (Kupiec, 2008 [1], p.26).

Pourquoi, dès lors, serait-il pertinent de faire remarquer à l'apprenant qu'il est un être vivant ? Pourquoi Kupiec, biologiste matérialiste, le fait-il toujours remarquer en situation de médiation scientifique, et pourquoi le faire en s'adressant à l'auditeur sur le plan émotionnel ?

Bachelard suggère que le caractère irrésistible des métaphores qui fonctionnent comme des raccourcis explicatifs antiscientifiques vient de ce qu'elles flattent plus que d'autres certains de nos désirs ou de nos besoins, certaines fonctions vitales de notre corps et de notre économie psychique ${ }^{18}$. La hâte avec laquelle nous embrassons le modèle d'intelligibilité programmiste semble effectivement manifester la présence d'un intérêt inconscient - source d'illusion - que la conscientisation critique doit aider à prendre en charge, afin que l'apprenant puisse dépasser ce biais en conquérant une compréhension plus objective de la réalité. Faire remarquer à l'apprenant qu'il est lui-même vivant (pendant un cours ou une conférence à propos des sciences du vivant) n'est donc pas une observation factuelle anodine: dans ce contexte bien particulier qu'est une situation d'objectivation du fonctionnement organique, un tel énoncé prend une dimension performative. Provoquer l'apprenant à faire mentalement l'aller-retour entre son expérience de vivant et la modélisation objective du vivant peut l'aider à relier les concepts présentés au problème qu'ils sont censés résoudre (autrement dit : à comprendre le coût théorique qu'il a fallu payer pour que le modèle explicatif soit possible). Faire sentir à l'apprenant qu'il est aussi un être vivant permet ainsi de le conscientiser au fait que de nombreuses «évidences» subjectives doivent être utilisées comme autant de repoussoirs pour problématiser les concepts afin de mieux les maîtriser - en voyant dans les erreurs qu'ils rectifient leurs conditions d'utilisation correcte.

\footnotetext{
17 « Dans la classe de chimie moderne comme dans l'atelier de l'Alchimiste, l'élève et l'adepte ne se présentent pas de prime abord comme de purs esprits. La matière elle-même ne leur est pas une raison suffisante de calme objectivité. Au spectacle des phénomènes les plus frappants, l'homme va naturellement avec tous ses désirs, avec toutes ses passions, avec toute son âme. On ne doit donc pas s'étonner que la première connaissance objective soit une première erreur. » (Bachelard, 2004 [20], p.65). Notons que, pour Bachelard comme pour Canguilhem, le couple conceptuel «obstacle» et «rupture» épistémologiques fonctionne indifféremment (comme ici) pour l'apprentissage et pour la découverte.

${ }^{18}$ Voir le chapitre « Libido et connaissance objective » (Bachelard, 2004 [20], p.219-250).
} 


\section{Souligner le fait que les processus d'apprentissage font partie intégrante de l'activité organique peut inscrire l'introduction aux problèmes éthico-politiques liés au vivant dans une relative continuité par rapport à la démarche scientifique de connaissance objective}

Les raisons positives de pratiquer la réflexivité en sciences du vivant que va apporter Canguilhem $n e$ sont pas en contradiction avec la conception bachelardienne de l'objectivité scientifique mais bien en continuité. Canguilhem réaffirme en effet sans aucune restriction les principes bachelardiens dans le cadre des sciences du vivant : «il n'est pas certain que le terme de «vie », pris absolument, ait sa place ailleurs que dans une problématique philosophique » (Canguilhem, 1994, [8], p.238). Pour lui comme pour Bachelard, la démarche d'objectivation - structurellement réductionniste - est constitutive de la scientificité même. Toutefois - comme Bachelard cherchant en quoi consiste le «rationalisme chimique» ou le «rationalisme électrique»-Canguilhem cherche en quoi la spécificité de l'objet des sciences du vivant impose à cet ensemble de disciplines scientifiques à la fois une méthode et une heuristique spécifiques. Il cherche en quoi consiste le "rationalisme biologique ${ }^{19}$. Or, pour Canguilhem, l'originalité décisive des sciences du vivant vient de ce que leur objet - l'organisme d'un être vivant (à quelque échelle que l'envisagent les différentes disciplines est fondamentalement le lieu d'une expérience, le foyer d'un vécu, un individu qui - même s'il n'est pas toujours «sentient» (capable de sentir) - perçoit toujours de manière active son environnement, c'est-à-dire qu'il constitue toujours ce dernier en un «milieu» propre dont il se vit comme le «entre».

Cette idée centrale est résumée dans le jeu de mot qui fait le titre d'une de ses œuvres maîtresses : ce qui fonde l'autonomie des sciences du vivant - ce qui constitue leur originalité mais aussi leur défi propres -, c'est que «la connaissance de la vie» (au sens du génitif objectif) porte sur un objet (l'organisme) capable de perception c'est-à-dire d'interaction intelligente avec son environnement (une adaptation, par opposition à l'interaction mécanique des machines). Or cette activité perceptive qui se trouve dans toute vie organique constitue une forme de «connaissance » (de découverte, de reconnaissance, d'appropriation) de leurs territoires par les êtres vivants. Ce qui permet à Canguilhem de conclure qu'il n'y a rien d'abusif à définir cette activité perceptive comme une réelle « connaissance de la vie » (au sens du génitif subjectif), c'est que la connaissance scientifique, qui est bien sûr le propre de l'humain, ne saurait pourtant avoir aucune autre origine que la continuité avec cette expérience vécue, cette interaction vitale. Ainsi :

« La connaissance consiste concrètement dans la recherche de sécurité par réduction des obstacles, dans la constructions de théories d'assimilation. Elle est donc une méthode générale pour la résolution directe ou indirecte des tensions entre l'homme et le milieu. [...] Il n'est pas vrai que la connaissance détruise la vie, mais elle défait l'expérience de la vie, afin d'en abstraire (par l'analyse des échecs) des raisons de prudence et des lois de succès éventuels, en vue d'aider l'homme à refaire ce que la vie a fait sans lui, en lui ou hors de lui. On doit dire par conséquent que, si pensée et connaissance s'inscrivent - du fait de l'homme - dans la vie pour la régler, cette même vie ne peut pas être la force mécanique, aveugle et stupide, qu'on se plaît à imaginer quand on l'oppose à la pensée. $[\ldots]$

Nous pensons, quant à nous, qu'un rationalisme raisonnable doit savoir reconnaître ses limites et intégrer ses conditions d'exercice. L'intelligence ne peut s'appliquer à la vie qu'en reconnaissant l'originalité de la vie. La pensée du vivant doit tenir du vivant l'idée du vivant. «Il est évident que pour le biologiste, dit Goldstein, quelle que soit

${ }^{19}$ Aussi appelé «rationalisme vital» (Delaporte, 1994 [21]) ou «vitalisme rationnel» (Morange, 2000 [22], p.92). 
l'importance de la méthode analytique dans ses recherches, la connaissance naïve (celle qui accepte simplement le donné) est le fondement principal de sa connaissance véritable et lui permet de pénétrer le sens des événements de la nature ». Nous soupçonnons que, pour faire des mathématiques, il nous suffirait d'être anges, mais pour faire de la biologie, même avec l'aide de l'intelligence, nous avons besoin parfois de nous sentir bêtes. » (Canguilhem, 2000 [7], p.10-13)

Si le premier paragraphe évoque très fortement l'épistémologie de Dewey (1938 [23]), ce n'est pas le cas du second - car Canguilhem retravaille ses intuitions en les confrontant aux principes de l'épistémologie bachelardienne. Pour Canguilhem, c'est seulement en partant de ce que les sciences $d u$ vivant disent d'objectif sur la réalité de la vie qu'on peut faire commencer le débat éthicopolitique sur les problèmes liés au vivant sur une base "rationnelle » - c'est-à-dire à la fois constructive et critique (l'un n'ayant aucune valeur rationnelle sans l'autre). Mais, à l'inverse, on s'empêcherait (de façon contre-productive) de sensibiliser l'apprenant au fait que les « questions » éthico-politiques dont le vivant fait l'objet sont de véritables problèmes (qui font réellement débat) si l'on présentait la démarche scientifique des sciences du vivant comme le «simple » fait d'expliquer le fonctionnement d'une machine très complexe.

Pour paraphraser la citation précédente, on pourrait dire que, dans sa conférence, Kupiec fait «se sentir bête » son public - au sens où l'entend Canguilhem, c'est-à-dire au sens de «vivant». Mais alors pourquoi le faire? Dans la dernière phrase, Canguilhem affirme que c'est en vertu d'un «besoin » qu'il faudrait - «pour faire de la biologie» - «se» sentir «parfois» vivant. Raison positive de pratiquer la réflexivité, ce besoin (de se sentir «bête») est à la fois théorique et pratique il répond en effet au «double défi » que nous avions avancé en introduction.

C'est un besoin théorique car - en plus de l'initiation à la démarche d'objectivation commune à toute science - l'initiation aux sciences du vivant implique une sensibilisation à la démarche heuristique qui est propre à ces sciences-là. Or, selon Canguilhem, l'opération heuristique qui est la plus propre aux sciences du vivant, c'est que le chercheur essaie d'intégrer à ses modèles (donc à sa connaissance objective « de » la vie) la perception interactive que développe l'organisme pour adapter son milieu et s'y adapter. Dans le cadre des sciences du vivant, la médiation scientifique ferait ainsi face - sur le plan théorique - à un défi redoublé par rapport à la médiation des sciences de la matière : initier aux sciences du vivant suppose en effet à la fois de réaffirmer le fait qu'elles relèvent de la même scientificité que les autres sciences - puisqu'elles pratiquent la même démarche d'objectivation - et de faire comprendre pourquoi ces sciences sont autonomes, pourquoi elles ne se réduisent pas à une application de la chimie ou de l'informatique. Bien sûr, les façons de présenter ensemble ce double aspect sont extrêmement nombreuses et diverses; notre objectif, dans cet exposé, est simplement de suggérer que la manière dont Canguilhem propose de le faire est originale dans la mesure où (comme on l'a vu chez Kupiec) elle consiste à conscientiser l'apprenant sur la base d'une réflexivité d'abord ressentie à la nécessité d'une vision scientifique du vivant.

Si «pour faire de la biologie » il faut se sentir «parfois » vivant, c'est aussi en vertu d'un besoin pratique (éthico-politique) répondant au second défi de l'éducation aux sciences du vivant. Cette raison reste implicite dans cet extrait, mais elle est la thèse centrale du Normal et le pathologique : il est impossible de décider du caractère pathologique ou normal d'un fait (IMC de la personne, résultat de prise de sang, anomalie anatomique, etc.) sans «passer par le point de vue » du patient, c'est-àdire sans chercher à savoir (en lui donnant la parole) dans quel mode d'existence - dans quelle relation au milieu - ce fait s'insère, fonctionne, fait sens. La thèse forte de Canguilhem est en effet que l'expérience du patient constitue un savoir - un savoir «vital » à tous les sens du terme puisque le diagnostic même ne peut pas décréter «anormal» tel ou tel état de fait sans l'avoir d'abord 
envisagé du point de vue de la façon de vivre du sujet ${ }^{20}$. Tout l'enjeu de la formation médicale est là : sous la formule « apprendre à articuler le cure (traitant la maladie) et le care (soignant la personne) », il s'agit de donner les moyens à l'apprenti-soignant de mettre en relation son savoir de professionnel de santé (savoir scientifique) et son savoir de patient (savoir d'expérience), mais pour qu'un tel rapprochement induise l'empathie (la capacité à envisager la situation du point de vue de l'autre) plutôt que l'émotion compassionnelle, il faut que la mise en relation se fasse sur une base rationnelle. Or il en va de même, mutatis mutandis, si l'éducation aux sciences du vivant doit introduire l'apprenant aux enjeux éthiques et politiques liés au vivant.

Le «besoin» de se sentir vivant «pour faire de la biologie ${ }^{21}$ n'est plus ici heuristique : il devient plutôt une métaphore exprimant la nécessité de garder à l'esprit que l'organisme qu'on est en train de manipuler (en cours d'expérimentation) perçoit et interagit - car, selon Canguilhem, oublier cela reviendrait à traiter l'objet d'étude comme autre chose qu'un vivant. L'enjeu pour Canguilhem est de montrer que les problèmes éthico-politiques liés au vivant ne peuvent pas être sans rapport avec ce que la science dit du vivant - tout simplement parce que, si c'était le cas, cela voudrait dire qu'ils sont sans rapport avec la réalité, avec «ce que c'est, concrètement, qu'un être vivant ». Pour l'éducation et la médiation scientifique, c'est là un vrai défi car il s'agit d'éviter un double écueil : d'une part la relativisation des questions bio-éthiques et politiques à « une simple affaire de convictions personnelles n'ayant rien à voir avec l'objectivité scientifique», et d'autre part la tendance à faire comme si les questions de société autour du vivant n'étaient qu'une affaire «de pédagogie » où il suffirait de « dépassionner » l'échange pour qu'enfin le bon sens s'aperçoive que les problèmes éthico-politiques se réduisent à des questions techniques.

Donner ponctuellement à l'apprenant l'occasion d'établir réflexivement un lien entre l'interaction incessante de l'organisme qu'il étudie avec son milieu et la capacité proprement humaine de simultanément cultiver un "point de vue » situé (subjectif) et adopter le «point de vue de nulle part» sur la réalité (vision objective progressivement construite par la science) : c'est, selon Canguilhem, un véritable moyen d'inscrire l'introduction aux problèmes éthico-politiques relatifs au vivant dans une relative continuité par rapport à la démarche scientifique de connaissance objective de la réalité.

Tout occupé à faire vivre ce débat à l'intérieur de son œuvre, à démontrer que cette "relative continuité » entre les problèmes éthico-politiques et l'effort de connaissance objective par la science est nécessaire comme un défi, Canguilhem n'a rien écrit sur les moyens concrets qui permettent ainsi de fonder le débat rationnel sur les résultats et la démarche des sciences du vivant. C'est plutôt bon signe : puisque la situation pédagogique est un moment de vie aussi bien pour l'apprenant que pour l'enseignant ou le médiateur, cela signifie que (pour Canguilhem) ce qu'il se passe entre eux relève comme dans le «spectacle vivant» - au moins autant de l'improvisation que de la préparation. Pour que l'apprenant ne se contente pas «d'intégrer » le savoir mais se l'approprie véritablement, il faut que quelque chose de performatif ait lieu : comme dans l'expérience de pensée proposée par Kupiec, l'apprenant doit réussir à réinvestir des représentations objectivées (modèles conceptuels abstraits) non pas seulement dans son effort pour vivre (comme chez Dewey) mais dans son effort réflexif pour "passer» du «dedans » au « dehors », du subjectif à l'objectif, du vécu organique à l'objectivation, $\mathrm{du}$ «point de vue situé» au «point de vue de nulle part». Pour Canguilhem, la performativité qui

\footnotetext{
${ }^{20}$ «Nous soutenons que la vie d'un vivant - fût-ce d'une amibe - ne reconnaît les catégories de santé et de maladie que sur le plan de l'expérience, qui est d'abord épreuve au sens affectif du terme, et non sur le plan de la science. La science explique l'expérience mais elle ne l'annule pas pour autant. » (Canguilhem, 1972 [6], p.131). ${ }^{21}$ Une expression qui signifie aussi bien étudier la biologie que travailler comme biologiste. Bachelard et Canguilhem insistent en effet sur la continuité des deux activités, afin de souligner le fait que toute démarche scientifique comporte un moment où chercheurs et apprenants doivent "problématiser » les concepts de la discipline (y compris les plus fondamentaux, les concepts paradigmatiques): revenir sur les conditions auxquelles ces concepts solutionnent leur problème de départ pour estimer le coût théorique au prix duquel la théorie a pu être mise sur pied. C'est ce pour quoi milite d'ailleurs aussi J.-J. Kupiec (2008 [1], p.57-77).
} 
permet à l'apprenant de s'approprier la démarche scientifique en sciences du vivant repose sur un moment corporellement vécu: le moment où nous nous sentons «bête», le vertige que nous ressentons en nous identifiant à l'une de nos gamètes, le malaise que nous vivons quand défilent sous nos yeux les planches d'anatomie pathologique. Tout cela constitue un savoir - un savoir de vivantet un savoir qui non seulement est utile négativement (pour conscientiser contre les projections illusoires, vitalistes et finalistes notamment), mais aussi positivement sur les plans heuristique et méthodologique (ou méta-cognitif). Ce savoir-là pourrait servir de point de départ aux discussions rationnelles des débats éthico-politiques à propos du vivant.

\section{Références}

1. J.-J. Kupiec, L'origine des individus (Fayard, Paris, 2008).

2. J.-J. Kupiec, L'ontophylogenèse : évolution des espèces et développement de l'individu (Quae, Versailles, 2012).

3. J.-J. Kupiec, M. Morange, Échelles, temporalités, causalité en biologie : quelles places pour l'individuel et le spécifique dans l'étude du vivant ?, in B. Durrive, J. Henry (dir.), Redéfinir l'individu à partir de sa trajectoire (p. 251-280) (Matériologiques, Paris, 2015).

4. M. Morange, Retour sur Le normal et le pathologique, in A. Fagot-Largeault, C. Debru (dir.), Philosophie et médecine : en hommage à Georges Canguilhem (p.167-169) (Vrin, Paris, 2008).

5. T. Nagel, The view from nowhere (Oxford University Press, New York, 1986).

6. G. Canguilhem, Le normal et le pathologique (PUF, Paris, 1972).

7. G. Canguilhem, La connaissance de la vie (Vrin, Paris, 2000).

8. G. Canguilhem, Études d'histoire et de philosophie des sciences concernant les vivants et la vie (Vrin, Paris, 1994).

9. J.-J. Kupiec (dir.), La vie, et alors? Débats passionnés d'hier et d'aujourd'hui (Belin, Paris, 2013).

10. J.-J. Kupiec, A probabilist theory for cell differentiation, embryonic mortality and DNA C-value paradox, Spec. Sc. \& Tech., 6, 471-478 (1983).

11. S. Freud, L'avenir d'une illusion (PUF, Paris, 1995).

12. F. Hallé, Éloge de la plante : pour une nouvelle biologie (Point, Paris, 1999).

13. J.-J. Kupiec, P. Sonigo, Ni Dieu ni gène. Pour une autre théorie de l'hérédité (Seuil, Paris, 2000).

14. S. Freud, Introduction à la psychanalyse (Payot, Paris, 1961).

15. R. Dawkins, The Selfish Gene (Oxford University Press, New York, 2006).

16. E. Husserl, La Terre ne se meut pas (Paris, Minuit, 1989).

17. S. Freud, Métapsychologie (Champs classiques, Paris, 2012).

18. G. Bachelard, Le rationalisme appliqué (PUF, Paris, 1966).

19. P. Bourdieu, Le métier de sociologue (Mouton de Gruyter, Berlin, 2005).

20. G. Bachelard, La formation de l'esprit scientifique (Vrin, Paris, 2004).

21. F. Delaporte, A vital rationalist: selected writings from G. Canguilhem (Zone Books, New York, 1994).

22. M. Morange, Georges Canguilhem et la biologie du $\mathrm{XX}^{\mathrm{e}}$ siècle, Rev. Hist. des Sc., 53-1, 38-106 (2000).

23. J. Dewey, Logic. The Theory of Inquiry (Holt \& co., New York, 1938). 